\title{
Anatomic and Functional Topography of the Dorsal Raphe Nucleus
}

\author{
JOLANE K. ABRAMS, PHILIP L. JOHNSON, JACOB H. HOLLIS, \\ AND CHRISTOPHER A. LOWRY \\ University Research Centre for Neuroendocrinology, University of Bristol, \\ Bristol BS2 $8 H W, U K$
}

\begin{abstract}
Serotonergic systems play an important and generalized role in regulation of sleep-wake states and behavioral arousal. Recent in vivo electrophysiologic recording studies in animals suggest that several different subtypes of serotonergic neurons with unique behavioral correlates exist within the brainstem raphe nuclei, raising the possibility that topographically organized subpopulations of serotonergic neurons may have unique behavioral or physiologic correlates and unique functional properties. We have shown that the stress-related and anxiogenic neuropeptide corticotropin-releasing factor can stimulate the in vitro neuronal firing rates of topographically organized subpopulations of serotonergic neurons within the dorsal raphe nucleus (DR). These findings are consistent with a wealth of behavioral studies suggesting that serotonergic systems within the DR are involved in the modulation of ongoing anxiety-related behavior and in behavioral sensitization, a process whereby anxiety- and fear-related behavioral responses are sensitized for a period of up to 24 to $48 \mathrm{~h}$. The dorsomedial subdivision of the DR, particularly its middle and caudal aspects, has attracted considerable attention as a region that may play a critical role in the regulation of acute and chronic anxiety states. Future studies aimed at characterization of the molecular and cellular properties of topographically organized subpopulations of serotonergic neurons are likely to lead to major advances in our understanding of the role of serotonergic systems in stress-related physiology and behavior.
\end{abstract}

KEYwORDS: fear; anxiety; anatomy; stress; serotonin; 5-hydroxytryptamine; 5-HT; topographic

\section{INTRODUCTION}

Serotonergic systems within the brainstem raphe complex are topographically organized with respect to their anatomic and functional properties. This anatomic and functional specificity raises the possibility that topographically organized subpopulations of serotonergic neurons may have unique stress-related functional properties. Although there are clear differences between the anatomic and functional properties of the dorsal raphe nucleus (DR) and the median raphe nucleus $(\mathrm{MnR}),{ }^{1}$ in this review we focus on the anatomic and functional topographic organization within the

Address for correspondence: Jolane K. Abrams, University Research Centre for Neuroendocrinology, University of Bristol, Marlborough Street, Bristol BS2 8HW, United Kingdom. Voice: +44 (0) 117-928-2181; fax: +44 (0) 117-928-2080.

e-mail: J.K.Abrams@bristol.ac.uk

Ann. N.Y. Acad. Sci. 1018: 46-57 (2004). () 2004 New York Academy of Sciences. doi: 10.1196/annals.1296.005 
DR, a brainstem raphe nucleus that contains an extensive group of serotonergic neurons (estimated to number 235,000 in the human brain ${ }^{2}$ ) projecting in a topographically organized manner to forebrain and brainstem targets. Recent technical advances have demonstrated that although the DR has been defined as a single nucleus by cytologic criteria, it comprises multiple subdivisions that differ with respect to the characteristics of individual serotonergic neurons found within them. Topographically organized subpopulations of serotonergic neurons within the DR differ in their morphologic features, membrane and cellular properties, afferent regulation, and efferent projection patterns. In this review, we evaluate whether or not these differences among subpopulations of serotonergic neurons have important implications for neural mechanisms underlying serotonergic modulation of stress-related physiology and behavior. In addition, we provide atlases of the rat and mouse DR and a proposed nomenclature that may prove valuable for future studies exploring the fine anatomic and functional topography of this nucleus.

\section{SEROTONERGIC NEURONS IN DIFFERENT SUBDIVISIONS OF THE DR HAVE UNIQUE MORPHOLOGIC AND CELLULAR FEATURES}

Serotonergic neurons in the rat DR appear in topographically organized groups of small round cells, medium-sized fusiform and bipolar cells, large fusiform cells, and very large multipolar cells. ${ }^{3}$ Similarly, serotonergic neurons in the human DR appear in topographically organized groups of round, ovoid, fusiform, and multipolar cells. ${ }^{2}$ Although we cannot predict functional properties of serotonergic cells based on morphologic features, the fact that serotonergic neurons with different morphologic features are topographically organized within different subdivisions of the DR suggests a potential link between structure and function. Recently developed and recently optimized technical approaches, such as whole-cell patch-clamp electrophysiologic studies in visualized brain slices, should allow us to begin to investigate structure-function relationships.

In addition to regional differences in the morphology of topographically organized subpopulations of serotonergic neurons, immunohistochemical co-localization studies have demonstrated that topographically organized subpopulations of serotonergic neurons co-express different neurotransmitters, neuropeptides, or other proteins. For example, recent studies have identified a subpopulation of serotonergic neurons within the dorsomedial subdivision of the DR ( $-8.00 \mathrm{~mm}$ bregma) that also expresses corticotropin-releasing factor $(\mathrm{CRF}) .{ }^{4}$ This same study revealed, consistent with previous studies, ${ }^{5}$ that this region of the DR projects to the central nucleus of the amygdala (CeA), a stress-related forebrain structure that is part of a distributed central autonomic control system. These observations, along with the high expression of neurokinin-1 immunoreactivity on neuronal fibers within the dorsomedial DR, suggest that this subdivision may play a role in serotonergic modulation of stress-related physiology and behavior in rats and in stress-related neuropsychiatric disorders in humans.

\section{EFFERENT PROJECTIONS ARISING FROM THE DR ARE TOPOGRAPHICALLY ORGANIZED}

The midbrain raphe nuclei, including the $\mathrm{DR}$ and the $\mathrm{MnR}$, are the main sources of serotonergic innervation of forebrain stress circuits. ${ }^{1}$ The most consistent finding 
in anatomic studies is that serotonergic neurons within the DR are organized topographically in the rostrocaudal plane. That is, serotonergic neurons located more rostrally project selectively to specific forebrain sites (e.g., the caudate putamen, substantia nigra, and virtually all neocortical regions), whereas serotonergic neurons located more caudally project to other forebrain sites (e.g., the septum, hippocampus, and entorhinal cortex ${ }^{3,6-8}$ ). Serotonergic neurons that project to the caudate putamen do not project to the hippocampus, and vice versa. ${ }^{9}$ These simple anatomic observations suggest that topographically organized subpopulations of serotonergic neurons have unique functional properties associated with modulation of specific forebrain systems.

\section{SEROTONERGIC NEURONS GIVE RISE TO COLLATERAL PROJECTIONS TO FUNCTIONALLY RELATED TARGETS}

The use of multiple retrograde tracers in a single animal has led to the proposal that not only are serotonergic neurons topographically organized within the midbrain and pontine raphe nuclei, but that individual serotonergic neurons give rise to collateral (branched) projections to functionally related targets, such as those involved in stress responses. ${ }^{1}$ These collateral serotonergic projections could allow temporally coordinated modulation of specific neural circuits, such as those implicated in central autonomic control, anxiety, and conditioned fear, which include the $\mathrm{CeA}$ and the paraventricular nucleus (PVN) of the hypothalamus. ${ }^{1}$ Collateral projections from serotonergic neurons innervating the CeA and PVN are located primarily in medial regions of the middle DR and in bilateral clusters of cells near the cerebral aqueduct more caudally. ${ }^{5,10}$ These double retrograde-tracing studies and others ${ }^{10-13}$ provide clear evidence for an anatomic topography within the DR and suggest that individual serotonergic neurons give rise to functionally related target structures, providing an anatomic basis for serotonergic modulation of distributed functional systems within the brain.

\section{AFFERENT PROJECTIONS INNERVATING THE DR ARE TOPOGRAPHICALLY ORGANIZED}

Different subdivisions of the DR receive afferent input from different sources. Retrograde and anterograde studies provide evidence for specific afferents to the ventral and dorsal parts of the middle ( $-8.00 \mathrm{~mm}$ bregma) DR, the rostral DR $(\sim-7.30 \mathrm{~mm}$ bregma), and the ventrolateral DR. ${ }^{14,15} \mathrm{~A}$ recent immunohistochemical study in rats found that CRF-ir fibers in the DR are organized along a rostral-to-caudal gradient and that CRF-ir fibers innervating different subdivisions of the DR have different proportions of excitatory versus inhibitory synaptic specializations, with the majority of synapses between CRF-ir terminals and dendrites in the dorsolateral DR being excitatory and the majority of those in the ventromedial region being inhibitory. ${ }^{16}$ Although the origin of the full complement of CRF-ir fibers innervating the DR is uncertain, a proportion of CRF-ir afferents arises from the CeA, suggesting that they may play an important role in differential modulation of topographically organized subpopulations of serotonergic neurons under stress-related conditions. ${ }^{17}$ 


\section{FUNCTIONAL TOPOGRAPHIC ORGANIZATION OF THE DR}

\section{Behavioral Correlates of Neuronal Activity}

Some of the strongest evidence for topographic organization of subpopulations of serotonergic neurons comes from studies combining electrophysiologic data with behavioral observations. Single-unit extracellular recording studies in cats suggested the existence of two different types of serotonergic neurons, designated type I and type II serotonergic neurons, based on behavioral correlates. Type I serotonergic neurons are active during waking arousal but show decreased activity during stages of quiet waking (QW) and slow-wave sleep (SWS) and cease firing during paradoxic sleep (PS) or rapid eye movement sleep. Type II serotonergic neurons are highly topographically organized and are found only in small numbers in bilateral columns of cells at the caudal interface of the dorsal and median raphe nuclei (reminiscent of the interfascicular dorsal raphe nucleus in the rat brain). Type II serotonergic neurons, like type I neurons, are active during waking arousal and QW but remain active during SWS and PS. ${ }^{18}$ Due to the overwhelming preponderance of type I serotonergic neurons, it is likely that most conclusions drawn from single-unit electrophysiology studies are drawn from random sampling of this population of cells.

A recent study sampling a large number of presumed serotonergic neurons within the DR using single-unit recording methods has classified them into six different subtypes based on their electroencephalographic and behavioral correlates in cats. ${ }^{19}$ Serotonergic neurons were classified within two large categories: (1) typical serotonergic neurons (e.g., similar to type I serotonergic neurons described previously) and (2) atypical serotonergic neurons. Among the typical and atypical serotonergic neurons, subpopulations were identified that may correspond to type I and type II serotonergic neurons described previously (TABLE 1). According to Sakai et al. ${ }^{19}$ type I-A and type I-B serotonergic neurons are evenly distributed in the DR, discharge regularly at a high rate during active waking and at progressively slower rates during SWS, and cease firing during SWS or PS (type I-A) or only during PS (type I-B); atypical type I-C serotonergic neurons are unevenly distributed in the DR (located predominantly within the ventral DR, similar to the atypical type II serotonergic neurons) and discharge regularly at a high rate during active waking but have sustained activity during SWS and PS. Other atypical serotonergic neurons (types II-A, II-B, and II-C) have unique topographic distributions within the DR. Type II-A neurons are located predominantly within the dorsomedial DR and show reductions in firing during QW and SWS; unlike typical serotonergic neurons, the level of $\alpha_{1}$-adrenergic receptor activation does not seem to be a major determinant of neuronal activity during SWS and PS. This study of a large sample of serotonergic neurons within the DR points toward a greater degree of functional heterogeneity of serotonergic neurons within the DR than had previously been suspected.

\section{Cellular Properties and Afferent Control Mechanisms}

Consistent with findings that the DR consists of multiple topographically organized subpopulations of serotonergic neurons with unique behavioral correlates, studies investigating the responses of serotonergic neurons to specific afferent signals (neurotransmitters, neuropeptides) have demonstrated that responses are dependent on the topographic location of the recordings. 


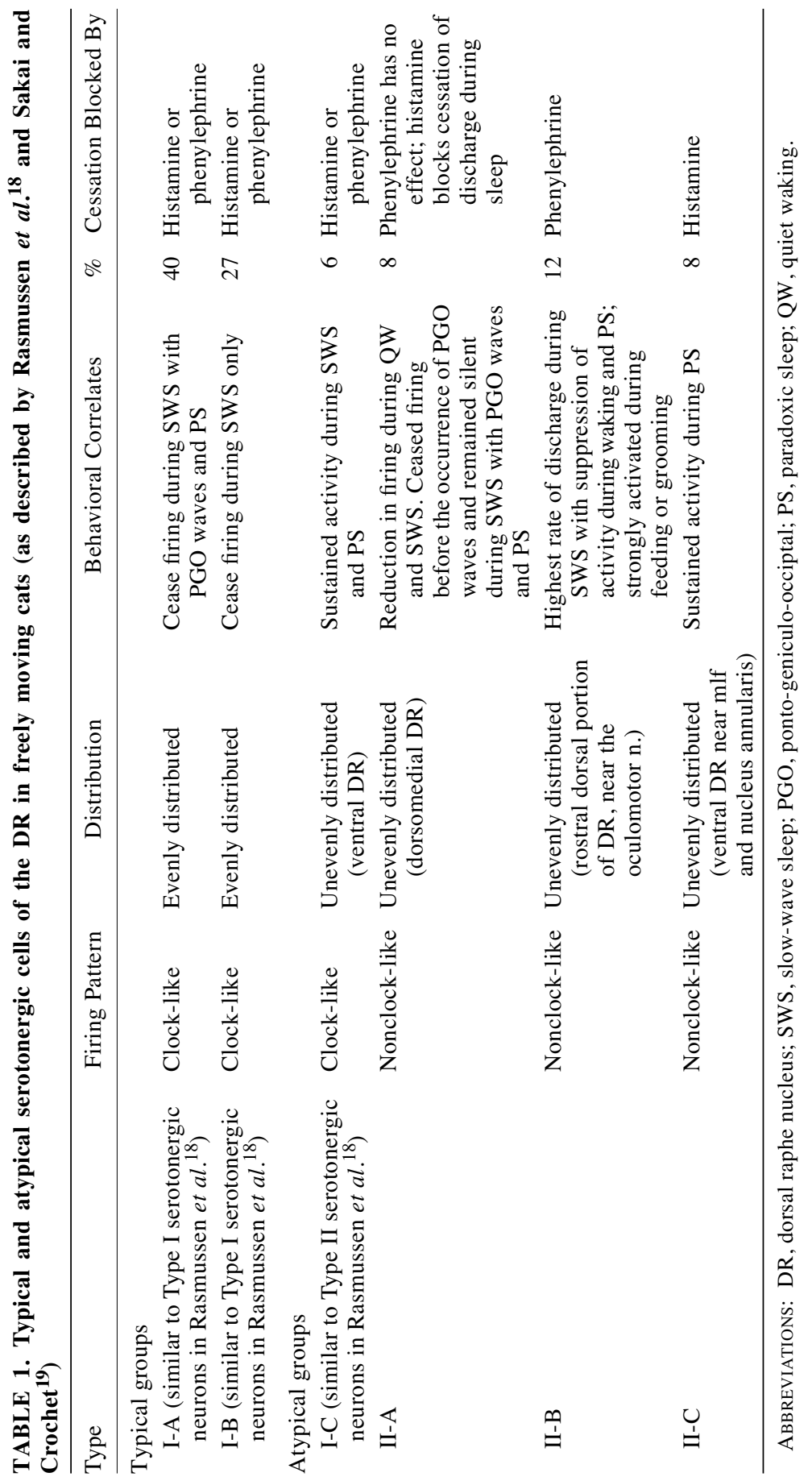


Electrophysiologic studies suggest that intracerebroventricular injection of CRF at low doses inhibits the firing rates of DR 5-HT neurons in vivo in a topographically dependent manner; the majority of the cells tested $(60.7 \%)$ in the ventromedial DR (-7.80 mm bregma) were inhibited by CRF, whereas responses of cells in the dorsomedial and lateral wings (ventrolateral $\mathrm{DR}$ ) were more variable. ${ }^{20,21}$ In vitro experiments in our laboratory have found a greater proportion of CRF-responsive neurons in the ventral midline of the caudal DR compared with the dorsal region of the DR at the same rostrocaudal level ( -8.00 to $-8.40 \mathrm{~mm}$ bregma $){ }^{22}$ Recent studies using visualized whole-cell patch-clamp electrophysiologic techniques reveal that serotonergic neurons within the dorsomedial and ventromedial DR differ with respect to active and passive membrane characteristics, ${ }^{23}$ further supporting distinctions among cellular properties and afferent control mechanisms of topographically organized serotonergic neurons within this nucleus.

Heterogeneity of 5-HT neuronal firing is also demonstrated in responses to compounds related to circadian fluctuations in behavioral arousal. The cessation of serotonergic neuronal firing in serotonergic neurons of the DR observed during PS is not the result of GABA-mediated inhibition ${ }^{24}$ but is rather the result of lack of facilitation resulting from the local release of norepinephrine and histamine. ${ }^{19}$ Different subtypes of serotonergic DR neurons respond differentially to microdialysis application of phenylephrine (an $\alpha_{1}$-adrenergic agonist) and histamine. Typical I-A and I-B neurons' normal quiescence during PS is blocked by phenylephrine or histamine, whereas the atypical II-A and II-C neurons' low firing rates are unaffected by phenylephrine but are totally (II-A) or partially (II-C) blocked by histamine (leading to substantial tonic activity during sleep). ${ }^{19}$ These results strongly suggest additional heterogeneity in the behavioral correlates and afferent control mechanisms of topographically organized subpopulations of serotonergic neurons within the DR.

\section{Behavioral Studies}

Anatomic and functional heterogeneity of the DR is nowhere more evident than in the modulation of stress-related neural circuits and stress responses. Behavioral studies involving site-specific injections of CRF into subregions of the DR support the hypothesis that the DR has a functional topographic organization in the rostrocaudal plane. A series of studies has demonstrated that increased serotonergic activity arising from the DR plays a critical role in the development of learned helplessness, including increases in behavioral measures of anxiety and fear measured 24 to $48 \mathrm{~h}$ after exposure to inescapable stress. ${ }^{25-27}$ Further studies have shown that these behavioral changes are dependent on CRF actions within the DR. Microinjection of the nonselective CRF receptor antagonist D-Phe-CRF $\mathrm{C}_{(12-41)}$ into specific sites in the caudal DR (particularly at $\sim-8.30 \mathrm{~mm}$ bregma) blocks behavioral changes induced by inescapable stress. ${ }^{28}$ Microinjections of CRF into the caudal DR (but not the rostral DR) are sufficient to induce learned helplessness $24 \mathrm{~h}$ later in the absence of any exposure to inescapable stress, ${ }^{28}$ consistent with the hypothesis that CRF actions on serotonergic systems arising from the caudal portion of the DR mediate these behavioral changes. This is consistent with the finding that exposure to inescapable stress increases the expression of the protein product of the immediate-early gene $c$-fos within the middle and caudal portions of the DR but not the rostral portion. This 
evidence points toward an essential role for the caudal DR in modulating the behavioral consequences of inescapable stress.

\section{IMMEDIATE-EARLY GENE EXPRESSION STUDIES}

There is a large body of data concerning the effects of stressful stimuli on the expression of immediate-early genes within the DR. ${ }^{29}$ Investigation of the effects of stress-related stimuli on the expression of the protein products of immediate-early genes such as $c$-fos is an effective means of evaluating the effects of a temporally defined stimulus on large populations of cells, with resolution of functional responses at the single-cell level. Paradigms associated with increased anxiety or conditioned fear, ${ }^{30-41}$ opiate withdrawal, ${ }^{42,43}$ and intracerebroventricular infusion of CRF or similar anxiogenic neuropeptides ${ }^{44,45}$ are effective at increasing immediate-early gene expression within caudal regions of the DR. When these data are taken together with those indicating increased serotonergic neurotransmission in the DR in response to stressful stimuli, ${ }^{46,47}$ especially when the stressor is intense, uncontrollable, or unpredictable, ${ }^{48-51}$ they suggest a mechanism whereby anxiogenic or fearinducing stimuli increase serotonergic neurotransmission via actions on subsets of serotonergic neurons within the caudal regions of the DR that give rise to a mesolimbocortical serotonergic innervation of the forebrain.

\section{THE NEED FOR DEFINITIVE NEUROANATOMIC MAPS}

With the goal of developing better treatments for human neuropsychiatric disorders in mind, it is essential that rostrocaudal levels of the DR and other raphe nuclei be carefully defined. A survey of the literature shows widely variable definitions, with some groups' definitions of "caudal" regions ${ }^{20,21}$ being almost as far forward as others" definitions of "rostral" ones ${ }^{22}$. Therefore, we propose consensus definitions based on standard stereotaxic atlases of the rat ${ }^{52}$ and mouse $\mathrm{e}^{53}$ brain and our own atlases of rat (FIG. 1) and mouse brain (FIG. 2) immunostaining with tryptophan hydroxylase.

According to these atlases, the "rostral" DR (regions associated with the caudal linear nucleus, ventrally) comprises anatomic levels from -6.92 to $-7.64 \mathrm{~mm}$ bregma in the rat brain ( -4.12 to $-4.48 \mathrm{~mm}$ bregma in the mouse); the "middle" DR includes anatomic levels from -7.73 to $-8.45 \mathrm{~mm}$ bregma in the rat brain $(-4.54$ to $-4.90 \mathrm{~mm}$ bregma in the mouse), and "caudal" DR includes anatomic levels from -8.54 to $-9.26 \mathrm{~mm}$ in the rat brain ( -4.96 to $-5.32 \mathrm{~mm}$ bregma in the mouse). We hope that these maps will prove useful in making meaningful comparisons among different studies of anatomic and functional subsets of serotonergic neurons in rodent brain.

FIGURE 1. Anatomic atlas of tryptophan hydroxylase (TrpOH) immunohistochemical (ir) staining in the rat DR. Sections $(30 \mu \mathrm{m})$ were obtained from an adult male Wistar rat, and every third section was immunostained with a sheep anti-TrpOH antibody. Bregma levels (mm) are indicated in each photograph. Scale bar represents $50 \mu \mathrm{m}$. Anatomic levels assigned based on comparisons with a rat stereotaxic atlas. ${ }^{52}$ 


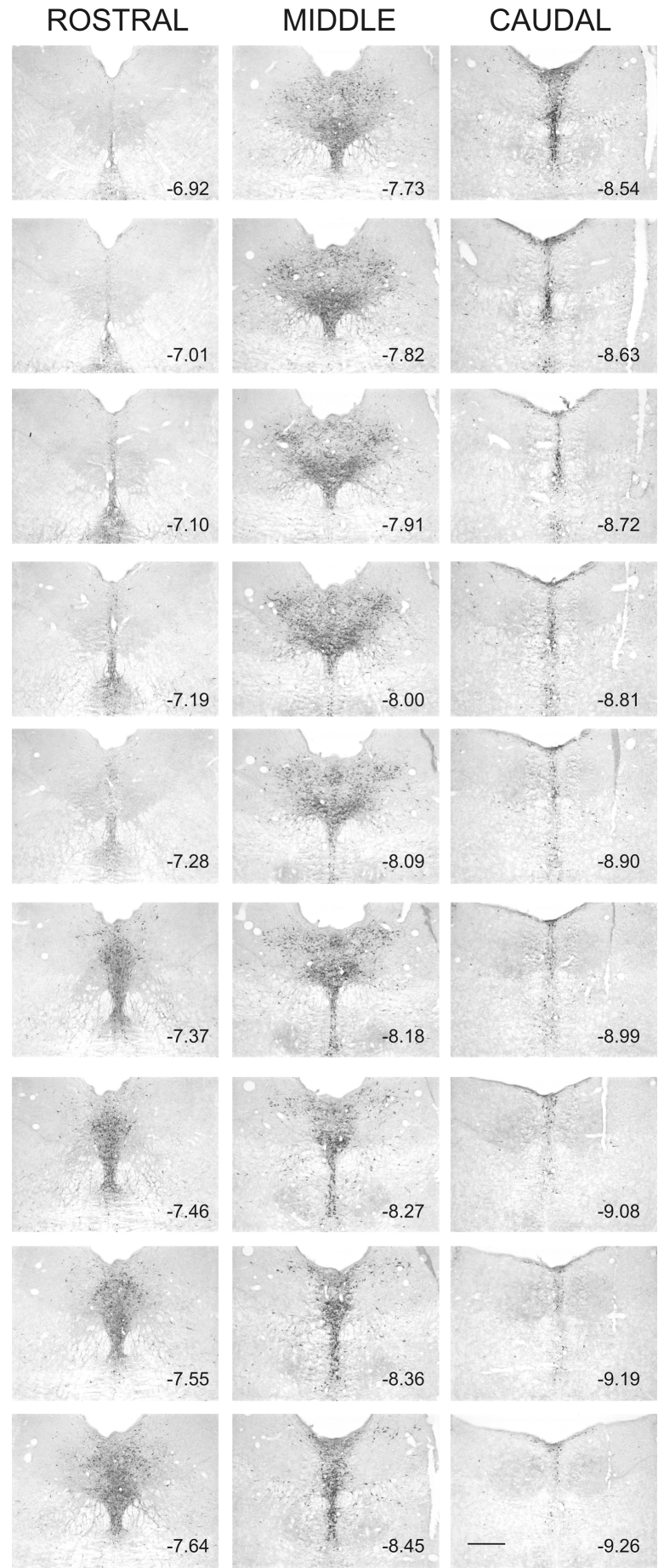




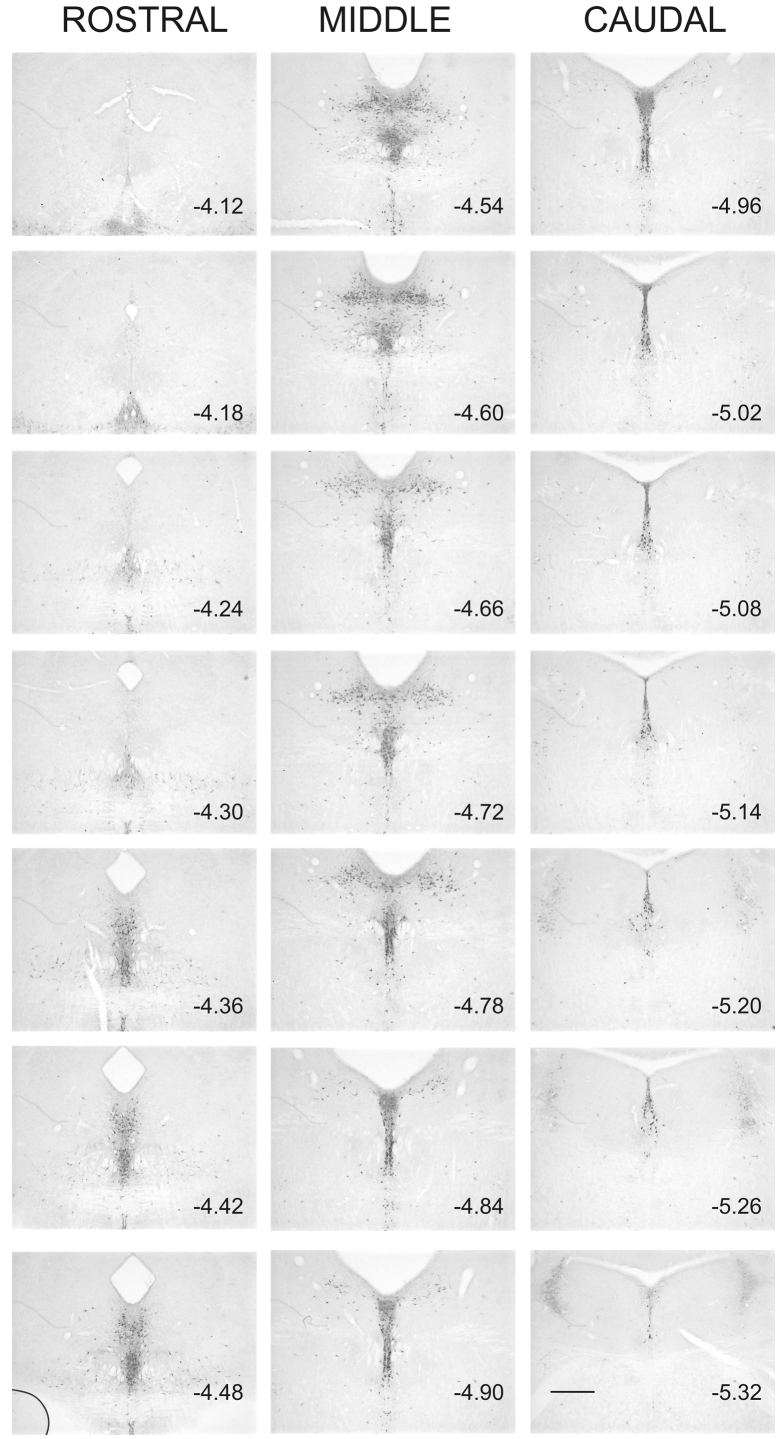

FIGURE 2. Anatomic atlas of TrpOH-ir staining in the mouse DR. Sections (30 $\mu \mathrm{m})$ were obtained from an adult male Balb/c mouse, and every second section was immunostained with a sheep anti-TrpOH antibody. Bregma levels $(\mathrm{mm})$ are indicated in each photograph. Scale bar represents $50 \mu \mathrm{m}$. Anatomic levels assigned based on comparisons with a mouse stereotaxic atlas. ${ }^{53}$ 


\section{ACKNOWLEDGMENTS}

We gratefully acknowledge the support of the BBSRC (7/S14666), the NIMH (R01MH065702-01A1), and the Neuroendocrinology Charitable Trust. C.A. Lowry is a Wellcome Trust Research Fellow.

\section{REFERENCES}

1. LOWRy, C.A. 2002. Functional subsets of serotonergic neurones: implications for control of the hypothalamic-pituitary-adrenal axis J. Neuroendocrinol. 14: 911-923.

2. BAKER, K.G., et al. 1990. Cytoarchitecture of the human dorsal raphe nucleus. J. Comp. Neurol. 301: 147-161.

3. SteinbUSCH, H.W. 1984. Serotonin-immunoreactive neurons and their projections in the CNS. In Handbook of Chemical Neuroanatomy, Vol. 3. A. Bjorklund \& T. Hokfelt, Eds.: 68-125. Elsevier. New York.

4. Commons, K.G., et al. 2003. A neurochemically distinct dorsal raphe-limbic circuit with a potential role in affective disorders. Neuropsychopharmacology 28: 206-215.

5. Petrov, T., et al. 1994. Chemically defined collateral projections from the pons to the central nucleus of the amygdala and hypothalamic paraventricular nucleus in the rat. Cell Tissue Res. 277: 289-295.

6. Steinbusch, H.W., et al. 1981. The nucleus raphe dorsalis of the rat and its projection upon the caudatoputamen: a combined cytoarchitectonic, immunohistochemical and retrograde transport study. J. Physiol. (Paris) 77: 157-174.

7. KoHLER, C., et al. 1982. Identification of serotonin and non-serotonin-containing neurons of the mid-brain raphe projecting to the entorhinal area and the hippocampal formation: a combined immunohistochemical and fluorescent retrograde tracing study in the rat brain. Neuroscience 7: 951-975.

8. VERTES, R.P. 1991. A PHA-L analysis of ascending projections of the dorsal raphe nucleus in the rat. J. Comp. Neurol. 313: 643-668.

9. ImAI, H., et al. 1986. The organization of divergent axonal projections from the midbrain raphe nuclei in the rat. J. Comp. Neurol. 243: 363-380.

10. Petrov, T., et al. 1992. The hypothalamic paraventricular and lateral parabrachial nuclei receive collaterals from raphe nucleus neurons: a combined double retrograde and immunocytochemical study. J. Comp. Neurol. 318: 18-26.

11. Villar, M.J., et al. 1988. Dorsal raphe serotoninergic branching neurons projecting both to the lateral geniculate body and superior colliculus: a combined retrograde tracing-immunohistochemical study in the rat. J. Comp. Neurol. 277: 126-140.

12. WiLSON, M.A., et al. 1991. The organization of serotonergic projections to cerebral cortex in primates: retrograde transport studies Neuroscience 44: 555-570.

13. LI, Y.Q., et al. 1993. Identification of periaqueductal gray and dorsal raphe nucleus neurons projecting to both the trigeminal sensory complex and forebrain structures: a fluorescent retrograde double-labeling study in the rat. Brain Res. 623: 267-277.

14. Peyron, C., et al. 1996. Lower brainstem catecholamine afferents to the rat dorsal raphe nucleus. J. Comp. Neurol. 364: 402-413.

15. Peyron, C., et al. 1998. Forebrain afferents to the rat dorsal raphe nucleus demonstrated by retrograde and anterograde tracing methods. Neuroscience 82: 443-468.

16. VAlENTiNO, R.J., et al. 2001. Evidence for regional heterogeneity in corticotropinreleasing factor interactions in the dorsal raphe nucleus. J. Comp. Neurol. 435: 450463.

17. SaKanaKa, M., et al. 1987. Corticotropin releasing factor-like immunoreactivity in the rat brain as revealed by a modified cobalt-glucose oxidase-diaminobenzidine method. J. Comp. Neurol. 260: 256-298.

18. Rasmussen, K., et al. 1984. Activity of serotonin-containing neurons in nucleus centralis superior of freely moving cats. Exp. Neurol. 83: 302-317.

19. SAKAI, K., et al. 2001. Differentiation of presumed serotonergic dorsal raphe neurons in relation to behavior and wake-sleep states. Neuroscience 104: 1141-1155. 
20. PRICE, M.L., et al. 1998. Effects of corticotropin-releasing factor on brain serotonergic activity. Neuropsychopharmacology 18: 492-502.

21. KIRBY, L.G., et al. 2000. Effects of corticotropin-releasing factor on neuronal activity in the serotonergic dorsal raphe nucleus. Neuropsychopharmacology 22: 148-162.

22. LOWRY, C.A., et al. 2000. Corticotropin-releasing factor increases in vitro firing rates of serotonergic neurons in the rat dorsal raphe nucleus: evidence for activation of a topographically organized mesolimbocortical serotonergic system. J. Neurosci. 20: $7728-7736$.

23. BECK, S.G. 2002. Median and dorsal raphe cellular characteristics: similarities and differences [abstract]. 2002 Abstract Viewer/Itinerary Planner. Society for Neuroscience, Washington, D.C. 636.4 .

24. SAKAI, K., et al. 2000. Serotonergic dorsal raphe neurons cease firing by disfacilitation during paradoxical sleep. Neuroreport 11: 3237-3241.

25. MAIER, S.F., et al. 1993. The role of the amygdala and dorsal raphe nucleus in mediating the behavioral consequences of inescapable shock. Behav. Neurosci. 107: 377388.

26. MAIER, S.F., et al. 1994. Chlordiazepoxide microinjected into the region of the dorsal raphe nucleus eliminates the interference with escape responding produced by inescapable shock whether administered before inescapable shock or escape testing. Behav. Neurosci. 108: 121-130.

27. MAIER, S.F., et al. 1995. 8-OH-DPAT microinjected in the region of the dorsal raphe nucleus blocks and reverses the enhancement of fear conditioning and interference with escape produced by exposure to inescapable shock. Behav. Neurosci. 109: 404-412.

28. Hаммаск, S.E., et al. 2002. The role of corticotropin-releasing hormone in the dorsal raphe nucleus in mediating the behavioral consequences of uncontrollable stress. J. Neurosci. 22: 1020-1026.

29. SENBA, E., et al. 1997. Stress-induced expression of immediate early genes in the brain and peripheral organs of the rat. Neurosci. Res. 29: 183-207.

30. Pezzone, M.A., et al. 1993. Activation of brainstem catecholaminergic neurons by conditioned and unconditioned aversive stimuli as revealed by c-Fos immunoreactivity. Brain Res. 608: 310-318.

31. Silveira, M.C., et al. 1993. Induction of Fos immunoreactivity in the brain by exposure to the elevated plus-maze. Behav. Brain Res. 56: 115-118.

32. BECK, C.H., et al. 1995. Conditioned fear-induced changes in behavior and in the expression of the immediate early gene c-fos: with and without diazepam pretreatment. J. Neurosci. 15: 709-720.

33. Matsuda, S., et al. 1996. Persistent c-fos expression in the brains of mice with chronic social stress. Neurosci. Res. 26: 157-170.

34. Beckett, S.R., et al. 1997. Central c-fos expression following $20 \mathrm{kHz} / \mathrm{ultrasound}$ induced defence behaviour in the rat. Brain Res. Bull. 42: 421-426.

35. CAmpeau, S., et al. 1997. Neuroendocrine and behavioral responses and brain pattern of c-fos induction associated with audiogenic stress. J. Neuroendocrinol. 9: 577-588.

36. Kollack-WalKer, S., et al. 1997. Social stress in hamsters: defeat activates specific neurocircuits within the brain. J. Neurosci. 17: 8842-8855.

37. Martinez, M., et al. 1998. Adaptation in patterns of c-fos expression in the brain associated with exposure to either single or repeated social stress in male rats. Eur. J. Neurosci. 10: 20-33.

38. NikUlina, E.M., et al. 1998. Behavioral sensitization to cocaine after a brief social stress is accompanied by changes in fos expression in the murine brainstem. Brain Res. 810: 200-210.

39. Chung, K.K., et al. 1999. Central serotonin depletion modulates the behavioural, endocrine and physiological responses to repeated social stress and subsequent c-fos expression in the brains of male rats. Neuroscience 92: 613-625.

40. GRAHN, R.E., et al. 1999. Activation of serotonin-immunoreactive cells in the dorsal raphe nucleus in rats exposed to an uncontrollable stressor. Brain Res. 826: 35-43.

41. Chung, K.K., et al. 2000. c-fos expression, behavioural, endocrine and autonomic responses to acute social stress in male rats after chronic restraint: modulation by serotonin. Neuroscience 95: 453-463. 
42. Chieng, B., et al. 1995. Increased fos-like immunoreactivity in the periaqueductal gray of anaesthetised rats during opiate withdrawal. Neurosci. Lett. 183: 79-82.

43. Chahl, L.A., et al. 1996. Distribution of c-Fos in guinea-pig brain following morphine withdrawal. Brain Res. 717: 127-134.

44. Vaughan, J., et al. 1995. Urocortin, a mammalian neuropeptide related to fish urotensin I and to corticotropin-releasing factor. Nature 378: 287-292.

45. BitTenCourt, J.C., et al. 2000. Do centrally administered neuropeptides access cognate receptors? An analysis in the central corticotropin-releasing factor system. J. Neurosci. 20: 1142-1156.

46. AdELL, A., et al. 1997. Comparative study in the rat of the actions of different types of stress on the release of 5-HT in raphe nuclei and forebrain areas. Neuropharmacology 36: 735-741.

47. MASWOOD, S., et al. 1998. Exposure to inescapable but not escapable shock increases extracellular levels of 5-HT in the dorsal raphe nucleus of the rat. Brain Res. 783: $115-120$.

48. AdELL, A., et al. 1988. Time course of changes in serotonin and noradrenaline in rat brain after predictable or unpredictable shock. Brain Res. 459: 54-59.

49. Inoue, T., et al. 1994. Regional changes in dopamine and serotonin activation with various intensity of physical and psychological stress in the rat brain. Pharmacol. Biochem. Behav. 49: 911-920.

50. Амат, J., et al. 1998. Escapable and inescapable stress differentially alter extracellular levels of 5-HT in the basolateral amygdala of the rat. Brain Res. 812: 113-120.

51. АмAт, J., et al. 1998. Escapable and inescapable stress differentially and selectively alter extracellular levels of 5-HT in the ventral hippocampus and dorsal periaqueductal gray of the rat. Brain Res. 797: 12-22.

52. Paxinos, G., et al. 1997. The Rat Brain in Stereotaxic Coordinates. Academic Press. San Diego, CA.

53. Paxinos, G., et al. 2001. The Mouse Brain in Stereotaxic Coordinates, 2nd Edition. Academic Press. San Diego, CA. 